\title{
Linear Regression of Eye Velocity on Eye Position and Head Velocity Suggests a Common Oculomotor Neural Integrator
}

\author{
MARK S. GOLDMAN, ${ }^{1}$ CHRIS R. S. KANEKO, ${ }^{2}$ GUY MAJOR, ${ }^{3}$ EMRE AKSAY, ${ }^{3}$ \\ DAVID W. TANK ${ }^{3,4}$ AND H. S. SEUNG ${ }^{1}$ \\ ${ }^{1}$ Howard Hughes Medical Institute and Brain and Cognitive Sciences Department, Massachusetts Institute of Technology, \\ Cambridge, Massachusetts 02139; ${ }^{2}$ Department of Physiology and Biophysics and Regional Primate Research Center, \\ University of Washington, Seattle, Washington 98195; ${ }^{3}$ Biological Computation Research Department, Bell Laboratories, \\ Lucent Technologies, Murray Hill, New Jersey 07974; and ${ }^{4}$ Departments of Molecular Biology and Physics, \\ Princeton University, Princeton, New Jersey 08544
}

Received 5 December 2001; accepted in final form 12 April 2002

\begin{abstract}
Goldman, Mark S., Chris R. S. Kaneko, Guy Major, Emre Aksay, David W. Tank, and H. S. Seung. Linear regression of eye velocity on eye position and head velocity suggests a common oculomotor neural integrator. J Neurophysiol 88: 659-665, 2002; 10.1152/jn.00993.2001. The oculomotor system produces eye-position signals during fixations and head movements by integrating velocity-coded saccadic and vestibular inputs. A previous analysis of nucleus prepositus hypoglossi (nph) lesions in monkeys found that the integration time constant for maintaining fixations decreased, while that for the vestibuloocular reflex (VOR) did not. On this basis, it was concluded that saccadic inputs are integrated by the nph, but that the vestibular inputs are integrated elsewhere. We re-analyze the data from which this conclusion was drawn by performing a linear regression of eye velocity on eye position and head velocity to derive the time constant and velocity bias of an imperfect oculomotor neural integrator. The velocity-position regression procedure reveals that the integration time constants for both VOR and saccades decrease in tandem with consecutive nph lesions, consistent with the hypothesis of a single common integrator. The previous evaluation of the integrator time constant relied upon fitting methods that are prone to error in the presence of velocity bias and saccades. The algorithm used to evaluate imperfect fixations in the dark did not account for the nonzero null position of the eyes associated with velocity bias. The phase-shift analysis used in evaluating the response to sinusoidal vestibular input neglects the effect of saccadic resets of eye position on intersaccadic eye velocity, resulting in gross underestimates of the imperfections in integration during VOR. The linear regression method presented here is valid for both fixation and low head velocity VOR data and is easy to implement.
\end{abstract}

\section{IN T R O D U C T I O N}

The oculomotor neural integrator is responsible for converting velocity-coded eye movement commands to the eye position signals seen in oculomotor motoneurons. These commands include eye velocity-coded saccadic commands and head velocity-coded vestibular signals involved in the vestibulo-ocular reflex (VOR). Traditionally, a single oculomotor neural integrator has been thought to be responsible for the conversion of all horizontal velocity commands (Robinson 1975, 1989).

Address for reprint requests: M. Goldman, Brain and Cognitive Sciences Dept., MIT, E25-210, 45 Carleton St., Cambridge, MA 02139. (E-mail: mark_g@mit.edu).
To test the common integrator hypothesis, Kaneko (1997, 1999) used ibotenic acid to create permanent lesions of the nucleus prepositus hypoglossi (nph). In one animal, eight lesions were administered over several months and later validated histologically. Two separate procedures were used to measure the time constants of integration involved in maintaining fixations and in performance of the VOR. The fixation integrator was evaluated by measuring the eye position of a head-fixed animal during spontaneous saccades in the dark and fitting the intersaccadic intervals with an exponential function. From these experiments, Kaneko (1997) concluded that the fixation integrator had been made leaky by the lesions. The VOR integrator was tested by rotating the animal sinusoidally in the dark and comparing the phase of the head velocity to the phase of a sinusoidal fit to the eye velocity. In the absence of saccades, the eye velocity should be sinusoidal and lead the head velocity if the integrator is leaky. Finding insignificant phase differences in the VOR experiments, Kaneko (1999) concluded that the VOR integrator was intact and must reside in a separate, non-lesioned brain area. This result was surprising because it contrasted with the results of pharmacological inactivation studies that suggested a single common integrator residing in the nucleus prepositus hypoglossi-medial vestibular nucleus (nph-mvn) complex (Cannon and Robinson 1987; Cheron and Godaux 1987; Cheron et al. 1986).

Here we re-analyze the data from which this "separate integrators" conclusion was drawn, using a single fitting procedure for both fixation and VOR data. The differential equation for the oculomotor integrator expresses the intersaccadic eye velocity as a linear combination of eye position and head velocity, plus a constant bias. We perform a linear regression to this relationship, using the eye position and head velocity data as independent variables and the eye velocity data as the dependent variable. For this procedure, the only difference between analyzing VOR and fixation data is the presence or absence of a non-zero head-velocity term in the oculomotor integrator equation. In the DISCUSSION, we compare this method to other methods (Mettens et al. 1994; Rey and Galiana 1993; Schneider et al. 2000) developed to fit eye data from an animal with an imperfector integrator performing saccades.

Our re-analysis of the Kaneko lesion data suggests that there is 
a single common neural integrator for saccadic and vestibular input rather than two anatomically separate integrators of these inputs. The previous separate integrators conclusion was based on a phase-shift fitting method that assumes that the intersaccadic eye velocity in a lesioned animal during sinusoidal VOR is also sinusoidal. However, for an animal with a lesioned fixation integrator, each saccade leads to a jump in intersaccadic eye velocity that decays exponentially (Mettens et al. 1994; Rey and Galiana 1993; Schneider et al. 2000). These jumps superimpose exponentially decaying transients on top of the underlying sinusoidal behavior, obscuring the underlying sinusoidal behavior. The sinusoidal fitting method neglects these exponential transients, which we show leads to the null result found previously (Kaneko 1999). In the DISCUSSION, we also explain why some other previous phase-shift analyses of the sinusoidal VOR in lesioned animals did find significant phase shifts, leading to the conclusion that there is a single common integrator.

\section{METHODS}

\section{Electrophysiology and data acquisition}

We re-analyze data from the experiments of Kaneko (1997, 1999), and the experimental methodology is described fully there. Briefly, the results presented here are from one macaque (Macaca mulatta) monkey that received a series of eight punctate $(180-700 \mathrm{~nL})$ unilateral injections of ibotenic acid in the nph on alternating sides over a span of 1150 days. Within 1 day after each injection, drift in the dark and VOR were recorded, with the exact time of recording depending on when the animal recovered enough to track a target spot. Additional recordings were obtained from a few minutes after the injection and at increasing intervals of hours, days, and weeks.

Eye-position traces were sampled every $1 \mathrm{~ms}$ for drift in the dark experiments and at 600 points per cycle for VOR experiments. The VOR was tested at $\pm 10 \mathrm{deg}$ of head rotation for frequencies ranging from 0.1 to $2.0 \mathrm{~Hz}$. We focus here on the $0.1-\mathrm{Hz}$ data for which the combined effects of the direct (velocity) pathway and eye plant can be neglected (see Fitting method, below). Eye-velocity traces were derived by taking differences of successive eye position points and then convolving with a square smoothing window with a width of $67 \mathrm{~ms}$. In performing the least-squares fit, $50 \mathrm{~ms}$ of data prior to the saccade and $200 \mathrm{~ms}$ of data following the saccade were removed to account for pre-saccadic abnormalities and post-saccadic drift, respectively. Saccades were identified by using a velocity or acceleration threshold and verified by visual inspection of the data. Between saccades, an additional velocity or acceleration threshold was applied to remove exceptionally noisy sections of data. Individual trial lengths ranged from 24 to $180 \mathrm{~s}$ for fixation trials and from 75 to $285 \mathrm{~s}$ for the $0.1-\mathrm{Hz}$ VOR trials focused on here. For completeness, in producing Fig. 3, we have chosen not to discard any trials analyzed. However, in several of the fixation trials, we observed abnormally frequent saccades with extended periods of displacement at extreme positions maintained by microsaccades. Trials in which this occurred, even after applying a noise threshold, produced outliers with low values of the inverse time constant $|k|$. In addition, some trials performed 1 day post-lesion displayed extreme values of $k$ or the velocity bias, $v_{\text {bias }}$.

\section{Fitting method}

FIXATIONS IN THE DARK. For an animal performing gaze fixations in the dark, we model the eye position as the output of a neural integrator of velocity commands

$$
\frac{\mathrm{d} E}{\mathrm{~d} t}=k E+v_{\text {bias }}+v_{\text {sacc }}
$$

Here, $E$ represents the eye position ( $\mathrm{deg}), \mathrm{d} E / \mathrm{d} t$ is the eye velocity $(\mathrm{deg} / \mathrm{s})$, and $v_{\text {sacc }}$ represents saccadic velocity commands $(\mathrm{deg} / \mathrm{s})$. When $k=v_{\text {bias }}=0$, this equation represents a perfect integration of velocity commands into eye position, $E=\int v_{\text {sacc }} \mathrm{d} t$. Defects in this integration are represented by the sensitivity of the eye velocity to eye position, $k$, and a velocity bias, $v_{\text {bias }}$.

Traditionally, Eq. 1 is regarded as a description of the time evolution of the eye position, E. Equation 1 is solved explicitly for $E$ for an arbitrary intersaccadic interval, giving an exponential leakiness $(k<$ $0)$ or instability $(k>0)$ in eye position of time constant $1 /|k|$ and with null position $E_{\text {null }}=-v_{\text {bias }} / k$. Data from individual intersaccadic intervals are then fit to this functional form using a nonlinear leastsquares fitting procedure.

We instead regard Eq. 1, for times away from saccades, as a linear relationship between the eye velocity, relabeled $v_{\mathrm{E}}$, and the eye position, with slope $k$ and $y$-intercept $v_{\text {bias }}$

$$
v_{E}=k E+v_{\text {bias }}
$$

We perform a linear least-squares fit to this relationship by comparing the time series of all intersaccadic eye positions (Fig. 1, top panels) to the time series of corresponding intersaccadic eye velocities (Fig. 1, middle panels: gray traces, eye velocity; black traces, best least-squares fit to Eq. 2). For a normal animal, there is negligible correlation between eye position and eye velocity, corresponding to a value of $k$ near zero (Fig. 1A, bottom panel: gray, data; black line, fit to data). For an animal with a significantly impaired integrator, however, there is a strong correlation between eye position and eye velocity, and $k$ is significantly different from zero (Fig. $1 B$ ).

In performing fits of eye position to an exponential function, the null position of the eye is often estimated by hand before fitting. For animals with strong velocity bias and significant nystagmus (Fig. 1C), this manual estimate can be difficult. Kaneko (1997), assuming a null eye position of $0 \mathrm{deg}$, estimated a time constant $1 /|k|$ of $<2 \mathrm{~s}$ for the data shown in Fig. $1 C$. The new fit suggests that the time constant is much larger $(1 /|k|=21 \mathrm{~s})$ and that the animal has a significant velocity bias $\left(v_{\text {bias }}=-1.2 \mathrm{deg} / \mathrm{s}\right)$.

SINUSOIDAL VOR. A lesioned oculomotor neural integrator receiving general head-velocity inputs requires a two-stage model of integration, with the first stage representing the neural integrator and the second stage representing an eye plant driven by both the output of the neural integrator and the direct (velocity) pathway (Precht 1974). However, for low-frequency sinusoidal VOR where the contribution of the direct pathway and eye plant are small, the neural integrator can be isolated and the system modeled by a single-stage integrator equation. We therefore focus our analysis of the VOR on lowfrequency data $(f=0.1 \mathrm{~Hz})$ where this approximation is reasonable. Because head velocity is proportional to frequency for a fixed amplitude of rotation, analysis at low frequencies additionally has the advantage of making the defects in the integrator, $k E$ and $v_{\text {bias, }}$ a relatively large fraction of the eye velocity.

For a single common integrator receiving head-velocity commands, the description of the intersaccadic eye velocity for a single integrator (Eq. 2) can be augmented by a term accounting for head velocity commands arriving from the vestibular system. For the sinusoidal VOR with head velocity $A \sin (\omega t)$, this gives

$$
v_{E}=k E+v_{\text {bias }}-G A \sin (\omega t)
$$

where $G(\mathrm{deg} / \mathrm{s})$ defines the gain of the VOR and the minus sign indicates that the command velocity is opposite in sign to the chair rotation. Here, we assume the vestibular input is in-phase with the negative of the chair-rotation velocity at angular frequency $\omega=2 \pi f$ (deg/s), where $f$ is the rotation frequency in hertz. In the RESULTS (Common vs. separate integrator models for fixations and VOR), we examine this assumption and in the DISCUSSION, we describe exten- 
A
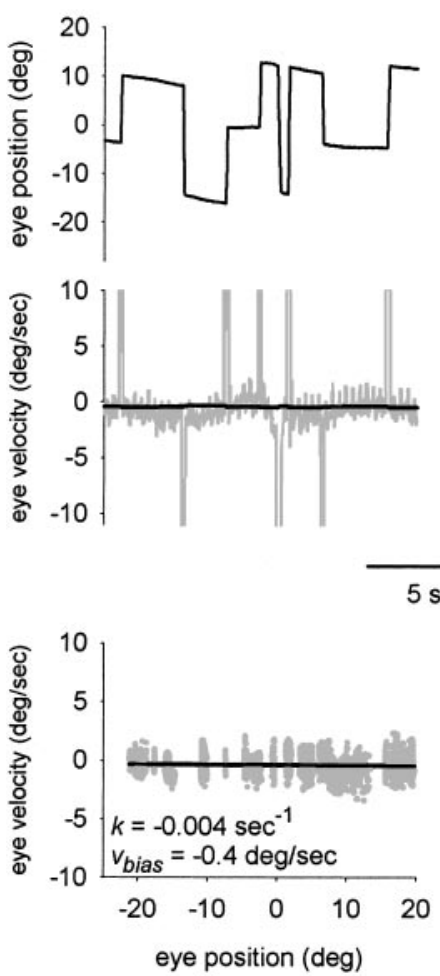

B
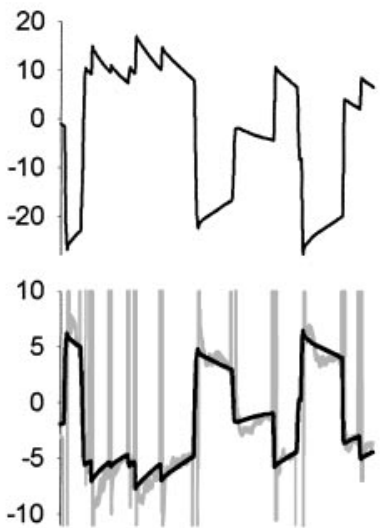

$5 \mathrm{sec}$

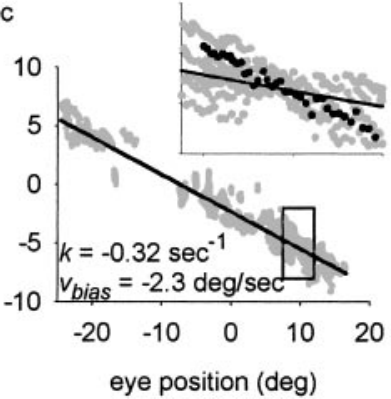

C
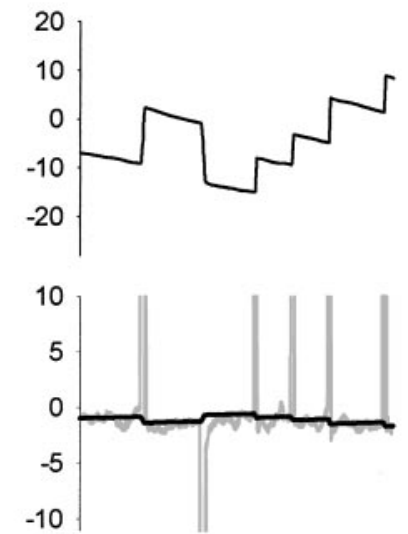

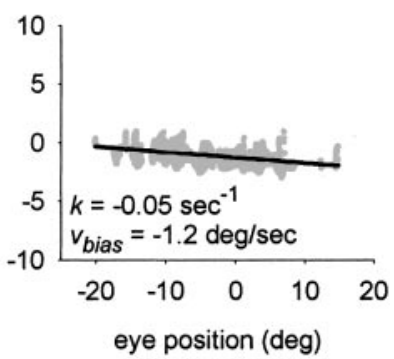

FIG. 1. Effect of lesions on fixations in the dark. A: eye velocity is independent of eye position prior to lesioning. top: horizontal eye position during fixations in the dark. middle: corresponding horizontal eye velocity (gray) with best least-squares fit (black line). Bottom: horizontal eye-drift velocity as a function of horizontal eye position (gray) and best least-squares fit to the data (black line, fit parameters: $\left.k=-0.004 \mathrm{~s}^{-1}, v_{\text {bias }}=-0.4 \mathrm{deg} / \mathrm{s}\right) . B$ : eye velocity is correlated with eye position following lesions [data shown: 55 days after the final (8th) lesion]. Top: horizontal eye position during fixations in the dark. Middle: corresponding horizontal eye velocity (gray dots) with best least-squares fit. Bottom: horizontal eye drift velocity as a function of horizontal eye position (black line, fit parameters: $k=-0.32 \mathrm{~s}^{-1}, v_{\text {bias }}=-2.3 \mathrm{deg} / \mathrm{s}$ ). Inset: magnified view of boxed region. The eye position and velocity data from a single intersaccadic fixation are highlighted in black. $C$ : example of an individual trial for which a traditional exponential fit is difficult (see text) (data shown: 51 days after the 2nd lesion). Top: horizontal eye position during fixations in the dark. Middle: corresponding horizontal eye velocity (gray dots) with best least-squares fit. Bottom: horizontal eye drift velocity as a function of horizontal eye position (black line, fit parameters: $k=-0.05 \mathrm{~s}^{-1}, v_{\text {bias }}=-1.2 \mathrm{deg} / \mathrm{s}$ ). Noise in the velocity traces is due to jitter (possibly microsaccades) observed during fixations and is less apparent in the more highly lesioned animals. In this and the following figures, positive eye position values indicate temporal eye positions and negative values indicate nasal eye positions.

sions to the model to account for higher order derivative terms or nonlinearities in eye position or head velocity.

We analyze Eq. 3 using the same linear regression procedure described in the previous section for fixations in the dark, now with the addition of the independent variable corresponding to head-velocity commands. The coefficients $k, G$, and $v_{\text {bias }}$ are derived by performing a linear least-squares fit of the intersaccadic eye velocity data to the eye-position data and the function $\sin (\omega t)$ (Fig. $2 A$ ). Although the fits are performed only at times away from saccades, the fits capture the effect of the saccades on the slow-phase eye velocity (gray line, Fig. 2A). Each time the animal saccades (clipped spikes in eye velocity trace, Fig. $2 A$ ), the eye-drift velocity jumps in the opposite direction, corresponding to a value of $k<0$. The linear regression fitting method captures these saccade-induced jumps in drift velocity because it explicitly models the changes in eye position that cause changes in the slow-phase eye velocity. Fitting a pure sinusoid to the data, as is often done (Kaneko 1999), neglects these jumps and consequently provides an extremely poor fit to the data (Fig. $2 B$, solid black trace).

Another common fitting technique, computing the phase shift between the peaks of the eye position and head position (or, equivalently, eye velocity and head velocity), is also prone to error because it neglects the effect of saccadic changes in eye position on the eye velocity. As demonstrated in Fig. $2 C$, the "phase difference" $\phi$ between peaks depends on the location of the eyes and whether the head position is at a peak or a trough. For eye positions less than the null position, there is a positive component to the eye velocity associated with leakiness of the integrator. This positive velocity causes the trough of the eye position to lead $(\phi>0)$ the trough of the command $(=-$ head) position at such positions (Fig. 2C, left pair of dashed lines: gray, trough of eye position; black, trough of head position). When the trough of the eye position occurs at a position more positive than the null position, the leakiness of the integrator causes the eye position trough to lag $(\phi>0)$ the trough of the command position (Fig. $2 C$, middle pair of dashed lines). When the eye position has a trough near the null position, there is little lag or lead between the troughs of the eye and command positions $(\phi \approx 0$; Fig. $2 C$, right pair of dashed lines).

\section{R E S U L T S}

\section{Comparison of $k$ and $v_{\text {bias }}$ for fixation and VOR trials}

We used the time-domain linear regression method (METHODS) to re-analyze the fixation and VOR experiments of Kaneko (1997, 1999). The resulting values for the sensitivity of 

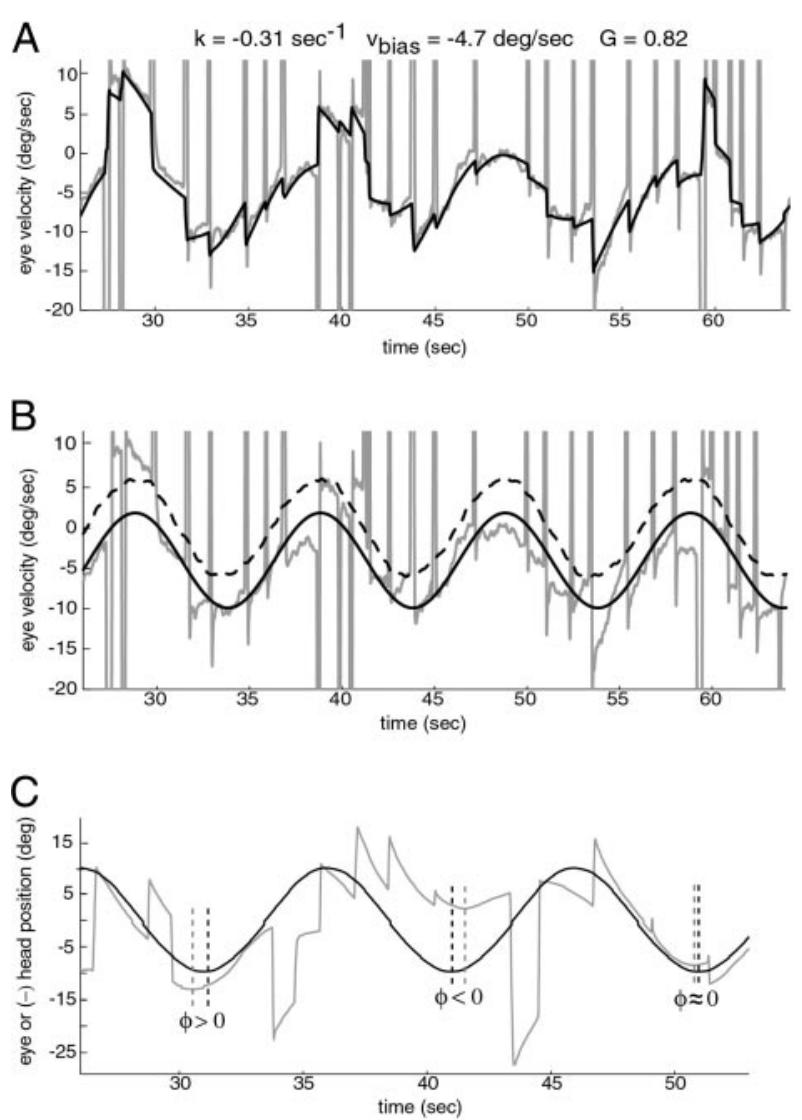

FIG. 2. Comparison of new fitting method to sinusoidal fitting methods. A: least-squares fitting method properly accounts for discontinuities in horizontal eye velocity following saccades in a lesioned animal ( 97 days following the 8th lesion). Gray line, horizontal eye velocity. Black line, least-squares fit to the model of $E q .3$ (fit parameters: $k=-0.31 \mathrm{~s}^{-1}, v_{\text {bias }}=-4.7 \mathrm{deg} / \mathrm{s}, G=0.82$ ). Saccadic spikes in eye velocity have been truncated. $B$ : horizontal eye velocity from the same data as in $A$ is poorly fit by a sinusoid (solid black line). Notice that the sinusoid cannot capture the discontinuities in eye-drift velocities that occur following saccades. Dashed black line shows the command $(=-$ head $)$ velocity. C: "phase difference" $\phi$ between peaks of head (black line) and eye (gray line) position depends on eye position in a lesioned animal (7 days following the 8th lesion, fit parameters: $k=-0.23 \mathrm{~s}^{-1}, v_{\text {bias }}=-1.6 \mathrm{deg} / \mathrm{s}$, $G=0.84)$ (see text).

eye velocity to eye position, $k$, and velocity bias, $v_{\text {bias }}$, are shown as a function of the number of days into the experiment and the number of lesions (Fig. 3). The values derived for fixations (black circles) and sinusoidal VOR (gray squares) overlap significantly, with a steady decay in the value of $k$ with an increasing number of lesions. The trial-averaged root mean square (rms) deviations in the fits to the intersaccadic eyevelocity data, computed for the trials following the final (8th) lesion, were comparable for the fixation and VOR experiments (fixations: rms deviation $=1.24 \pm 0.32 \mathrm{deg} / \mathrm{s}$; VOR: rms deviation $=1.31 \pm 0.16 \mathrm{deg} / \mathrm{s})$.

The results presented in Fig. 3 differ from the previously published analyses (Kaneko 1997, 1999) that reported no impairment of the integrator for VOR and found an immediate drop in $k$ for fixation trials following the first lesion (with most of these trials reporting $k<-0.2 \mathrm{~s}^{-1}$ ) that was maintained throughout the subsequent lesions. The differences can be attributed to systematic difficulties in the methodology used in the previous study (Figs. $1 C$ and $2 B$; and see Fitting method,
METHODS). The results of the re-analysis are consistent with a single common integrator for fixations and VOR.

\section{Common versus separate integrator models for fixations and} VOR

We next tested the hypothesis that the data would be better fit by a model in which the VOR is integrated by an unlesioned, anatomically separate area from the one responsible for saccades (Kaneko 1999). This was done by comparing the fits achieved by a separate integrators model with those found in the previous section for a single common integrator model (Eq. 3).

To model anatomically separate integrators for saccades and VOR, we assume that the eye position signal consists of independent contributions from the saccadic (fixation) integrator and the VOR integrator. For times away from saccades, this gives

$$
\begin{gathered}
E=E_{\text {fix }}+E_{\mathrm{VOR}} \\
\frac{\mathrm{d} E_{\mathrm{fix}}}{\mathrm{d} t}=k_{\mathrm{fix}} E_{\mathrm{fix}}+v_{\mathrm{fix}} \\
\frac{\mathrm{d} E_{\mathrm{VOR}}}{\mathrm{d} t}=-G_{\mathrm{VOR}} A \sin (\omega t)
\end{gathered}
$$

Here $k_{\text {fix }}$ and $v_{\text {fix }}$ are the position sensitivity and velocity bias of the lesioned fixation integrator, respectively, and $G_{\mathrm{VOR}}$ is
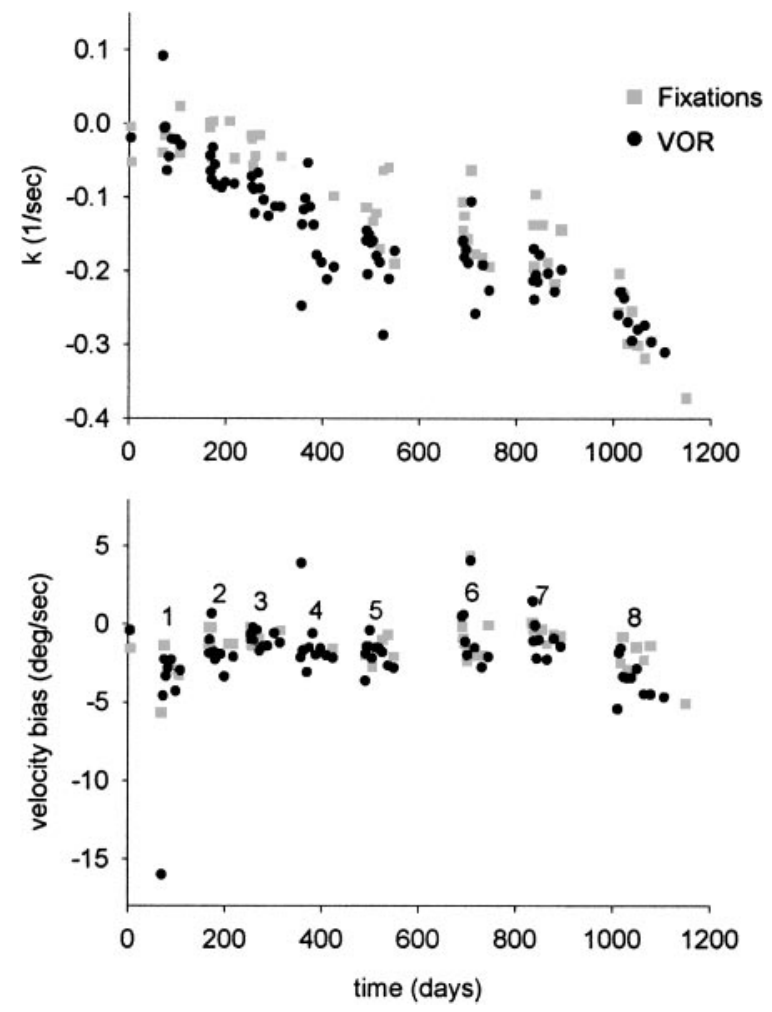

FIG. 3. Comparison of $k$ and $v_{\text {bias }}$ derived from fixations in the dark and from the VOR protocol. Velocity bias $v_{\text {bias }}$ (top) and inverse time constant $k$ (bottom) derived from least-squares fit to eye velocity for fixations in the dark (gray squares) and the VOR protocol (black circles). The $x$-axis is the number of days from the start of the experiment. The lesion number is marked in the lower panel, above the clusters of data points. Notice that the derived value of $k$ for both fixations and the VOR declines steadily with increasing numbers of lesions, suggesting that the integrators for fixations and VOR are affected equally. 
the gain of the VOR integrator. We approximate the unlesioned VOR integrator as being perfect $\left(k_{\mathrm{VOR}}=v_{\mathrm{VOR}}=0\right)$. Adding Eqs. 5 and 6, using Eq. 4 to re-write $E_{\text {fix }}$ in terms of $E$ and $E_{\mathrm{VOR}}$, and integrating $E q$. 6 to explicitly express $E_{\mathrm{VOR}}$ as a function of time gives

$$
\frac{\mathrm{d} E}{\mathrm{~d} t}=k E+v_{\text {bias }}-G_{\mathrm{VOR}} A \sin (\omega t)-B A \cos (\omega t)
$$

or equivalently

$$
\frac{\mathrm{d} E}{\mathrm{~d} t}=k E+v_{\text {bias }}-G A \sin \left(\omega t+\phi_{\mathrm{fit}}\right)
$$

where $k=k_{\mathrm{fix}}, B=k G_{\mathrm{VOR}} / \omega, G=\sqrt{G_{\mathrm{VOR}}^{2}+B^{2}}, \phi_{\mathrm{fit}}=\tan ^{-1}$ $(k / \omega)$, and $v_{\text {bias }}=v_{\text {fix }}$ plus a constant that depends on the initial condition of $E_{\mathrm{VOR}}$. Thus, the model for two separate integrators $(E q .8)$ is equivalent to a single integrator model (Eq. 3) with head-velocity commands phase-shifted by $\phi_{\text {fit. }}$. We use the notation $\phi_{\text {fit }}$ to indicate that we treat $\phi_{\text {fit }}$ as a free-fitting parameter that should assume a value near 0 deg if the eye position data are described by a single common integrator and should assume a value near $\tan ^{-1}(k / \omega)$ if the eye position data are described by the two separate integrators model outlined in Eqs. 4-6 above. For the two separate integrators model with $\omega=2 \pi(0.1 \mathrm{~Hz})$, when $k=-0.3$ [typical of the value for the fixation integrator after the final lesion (Fig. 3)], $\phi_{\text {fit }}=-25.5$ deg. We note that $\phi_{\text {fit }}$ is distinct from the phase shift $\phi$ between the command velocity and purely sinusoidal fit to the eye velocity used in the phase-shift methods portrayed in Fig. 2, $B$ and $C$.

Figure 4 shows the result of fitting the final (8th) lesion data to this model with $\phi_{\text {fit }}$ as a free parameter, or with $\phi_{\text {fit }}$ constrained to equal $\tan ^{-1}(k / \omega)$ (the separate integrators prediction). The actual fit was performed using Eq. 7, enabling the use of the linear least-squares fitting method (now with the addition of the extra parameter $B$ ). When left as a free parameter, $\phi_{\text {fit }}=6.2 \pm 1.8 \mathrm{deg}$ when averaged across trials, much closer to the single integrator prediction $\left(\phi_{\text {fit }}=0 \mathrm{deg}\right)$ than to the average separate integrators prediction $\left(\phi_{\mathrm{fit}}=-23.6 \mathrm{deg}\right)$. The separate integrators model $\left(\phi_{\text {fit }}\right.$ constrained to equal $\tan ^{-1}$ $(k / \omega)$, with $k$ taken from the fixation data) produces values of $k$ consistent with the unconstrained and constrained (Fig. 2A) common integrator models, but the fits produced by this model are visibly worse (Fig. $4 B$ ). The trial-averaged rms deviation for the unconstrained, constrained common integrator, and constrained separate integrators models are, respectively, 1.25, 1.31, and $2.20 \mathrm{deg} / \mathrm{s}$ (Fig. 4C). The above results suggest a single common integrator for fixations and VOR.

Previous studies of the phase shift of second-order vestibular neurons that provide input to the oculomotor neural integrator show a small deviation from a pure velocity input, corresponding to $\phi_{\text {fit }}>0$ (Fuchs and Kimm 1975). The small, positive phase shift $\phi_{\text {fit }}$ systematically found in the fit to the unconstrained model (Eq. 8) is consistent with this observation.

\section{Failure of traditional VOR methods; analysis of saccades}

In the absence of saccades, a leaky integrator receiving sinusoidal velocity commands generates a sinusoidal eye velocity trace that phase leads the command velocity by $\tan ^{-1}$ $(k / \omega)$. Kaneko (1999) noted that the data presented here generally display no phase shift between the command velocity
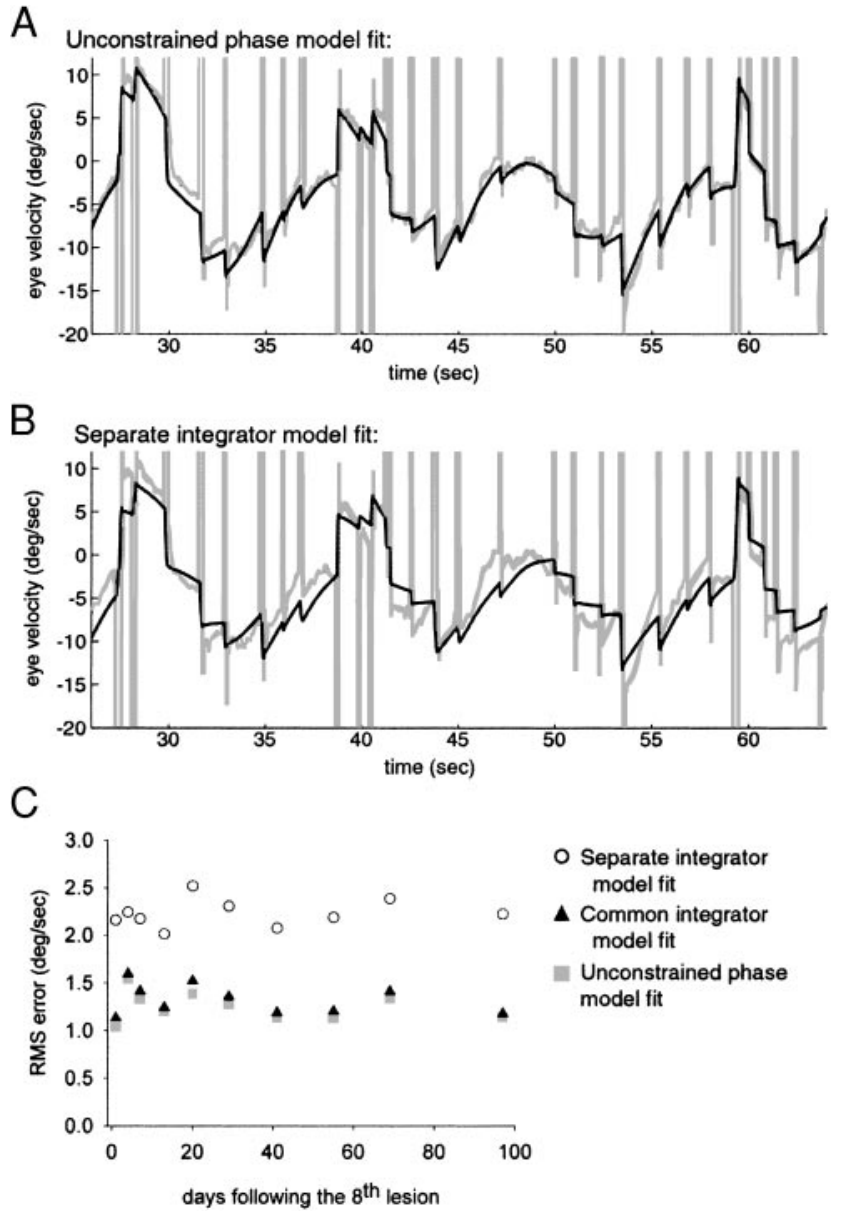

FIG. 4. The constrained separate integrators model performs a poor fit to the horizontal eye velocity data. $A$ : fit of the same horizontal eye velocity trace shown in Fig. $2 A$ (gray line) to a model with $\phi_{\text {fit }}$ left as a free parameter. The best fit (black line) occurs for $\phi_{\text {fit }}=4.4 \mathrm{deg}$, which is closer to the common integrator prediction $\left(\phi_{\text {fit }}\right.$ constrained to equal $0 \mathrm{deg}$ ) than to the separate integrators prediction $\left(\phi_{\text {fit }}\right.$ constrained to equal $-26.8 \mathrm{deg}$ ). Other fit parameters: $v_{\text {bias }}=-4.7 \mathrm{deg} / \mathrm{s}, k=-0.32 \mathrm{~s}^{-1}, G=0.82$. The fit is nearly indistinguishable from the fit to the common integrator model of Fig. $2 A$. B: fit of the horizontal eye velocity to a separate integrators model $\left(\phi_{\mathrm{fit}}\right.$ constrained to equal $-26.8 \mathrm{deg}$ ). The fit is much poorer than the fit to the common integrator model of Fig. $2 A$. $C$ : rms deviations of the least-squares fits to the horizontal eye velocity. Data taken after the final (8th) lesion was fit to the unconstrained phase (gray squares), common integrator (black triangles), and separate integrators (open circles) models. The common integrator model performs nearly as well as the unconstrained phase model. The separate integrators model fits the data considerably less well.

(Fig. 2B, dashed line) and a sinusoidal fit to the eye velocity (Fig. $2 B$, solid black line). Closer inspection of Fig. $2 B$ (gray eye velocity trace) shows why: the saccades (Fig. $2 B$, spikes in velocity) occur preferentially near the peaks of and in the opposite direction as the command velocity, causing jumps in the eye-drift velocity at these times (most easily seen for the less frequent negative-velocity spikes in Fig. $2 B$ ). These jumps at the peaks of the command velocity reset the phase shift accumulated during the slow phases, confounding the sinusoidal fitting method.

We analyzed the time series of saccade magnitudes and directions for the pre-lesion data and for all trials following the final lesion. Averaging across the lesion trials, the number of saccades occurring in a given direction had peaks that approximately coincide with the peaks of head velocity, with the 
probability of making a saccade in the positive or negative direction approximately equal to a term proportional to head velocity plus a constant offset reflecting the excess of saccades in the direction opposite the velocity bias (Fig. 5A, top). The resulting average saccade displacement for a given phase of the command $(=-$ head $)$ velocity likewise has peaks approximately coinciding with the peaks of head velocity that reflect the increased frequency of saccades in the same direction as the head velocity (Fig. 5A, bottom). Individual trials were noisier, but all trials show a large peak in the power spectrum of the time series of saccade sizes at the tested frequency of the VOR (Fig. 5B), indicating the periodic variation of saccade frequency and direction with head velocity. The variation of saccade frequency and direction with head velocity occurs in normal animals as well (Fig. 5C and Galiana 1991; Galiana and Outerbridge 1984) and may reflect re-centering and/or anticipatory movement of the animal's eyes to compensate for head rotations (Robinson 1981). It also has been observed in goldfish with both leaky and unstable integrators, where it similarly resulted in a resetting of the sinusoidal fitting method phase shift to a value near zero (G. Major, E. Aksay, R. Baker, and D. W. Tank, goldfish experiments, unpublished observations).

\section{I S C U S S I O N}

We have presented a new method for fitting the time constant and velocity bias of the oculomotor integrator. The method relies on a linear regression to the differential equation for the integrator and is applicable to both fixation data and VOR data taken at low head velocities to isolate the contribution of the neural integrator from that of the direct pathway and eye plant. Because the method uses linear regression of eyevelocity data on eye position and head-velocity data, it is easy to implement.

Traditional phase-shift analyses of the sinusoidal VOR implicitly assume that the intersaccadic eye velocity is sinusoidal. For an animal with a lesioned fixation integrator performing saccades, this assumption is not valid. When a saccade causes a jump in the eye position, it correspondingly causes a jump in the component of intersaccadic eye velocity associated with failure to maintain fixations. These jumps cause the eye velocity to deviate from the sinusoid predicted for a lesioned animal performing no saccades. Because the deviation decays away exponentially with the time constant of the fixation integrator, the eye velocity of an animal that saccades more frequently than the fixation integrator time constant cannot be reasonably approximated by a sinusoidal model that neglects the effect of saccades. However, if the time constant of integration $\tau=1 /|k|$ is reduced to values much lower than a typical intersaccadic interval, the eye velocity at times several time constants removed from the previous saccade should appear sinusoidal and the results of a phase-shift analysis may be at least qualitatively correct. This may explain why previous pharmacological inactivation studies (Cheron and Godaux 1987; Cheron et al. 1986) that reported much smaller time constants than those found here did find large phase shifts between command and eye velocities (or, equivalently, positions) and therefore concluded correctly that the VOR had been compromised.

Laplace transform methods have been developed that successfully deal with the transients introduced by saccades (Mettens et al. 1994; Rey and Galiana 1993; Schneider et al. 2000).
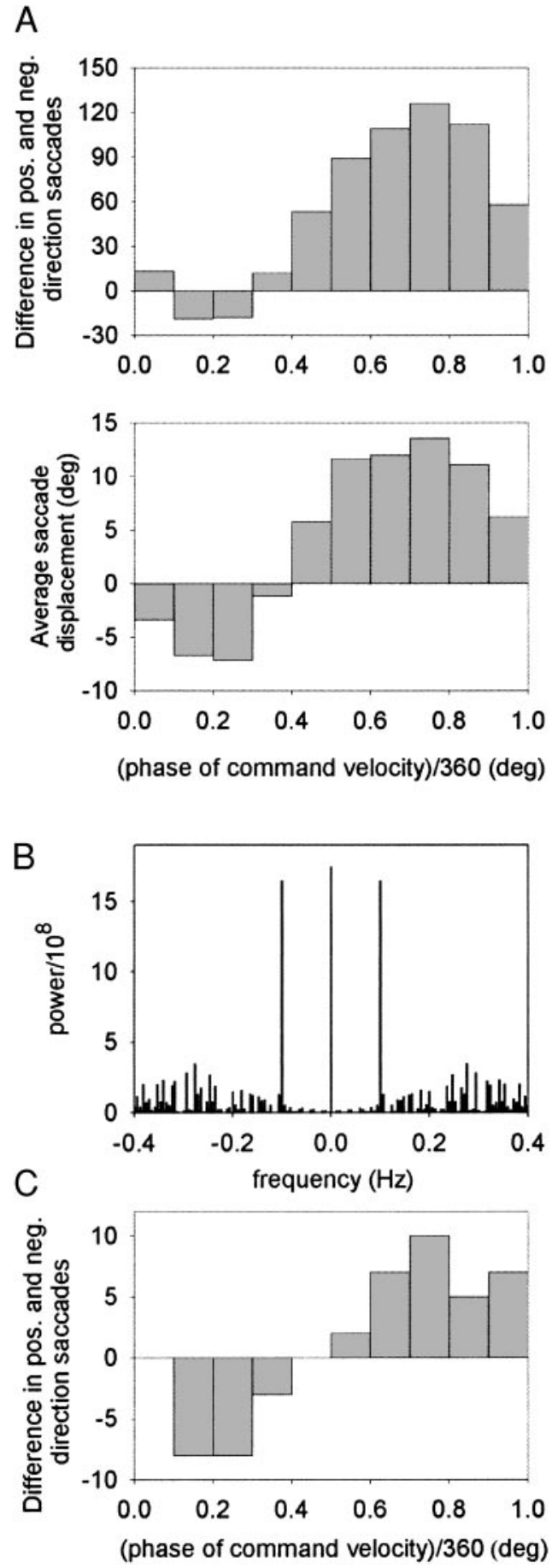

FIG. 5. Saccade magnitude, direction, and frequencies of occurrence are correlated with head velocity. $A$ : difference in the number of saccades occurring in the positive and negative directions (top) and average saccade displacement (bottom) as a function of the phase of the command ( = - head) velocity, where command velocity $=\sin (\omega t)$. Saccades occur preferentially on the peaks of head velocity and in the same direction as the head movement. Data shown reflect a sum of the 10 experiments performed after the final lesion. $B$ : power spectrum of the time series of saccade displacements during $0.1-\mathrm{Hz}$ VOR. The large peak at 0 frequency indicates the excess of ON-direction saccades across trials and reflects compensation for velocity bias. The large peaks at $0.1 \mathrm{~Hz}$ reflect correlations of the saccade frequencies of occurrence and amplitudes with head velocity. Data shown are from 97 days after the final lesion. $C$ : difference in the number of saccades occurring in the positive and negative directions as a function of the phase of the command velocity for the same animal prior to lesions. Data shown reflect a single experiment. 
These methods, in their simplest form, fit the Laplace transform of Eq. 8. In contrast to the methods presented here, they require a nonlinear parameter fitting procedure and, in some cases, invoke $n$ local fitting parameters (beyond the global parameters being extracted), one for each of the $n$ slow-phase segments being fit. The method presented here is linear, affording easy implementation, and global, allowing an entire set of data to be fit without invoking local parameters for each intersaccadic interval. For long trains of eye movement data with many intersaccadic intervals, this provides a sharp reduction in the number of fitting parameters, increasing confidence in the global parameters that are extracted. A potential disadvantage of the linear regression method is that the computation of the eye velocity is subject to noise. Although not encountered in this study, this could be a problem for highly noisy eye position data that cannot be sufficiently smoothed over a time scale that is small relative to those of interest.

Because the method presented here is a linear regression to the various terms of a differential equation, it may be extended to include different testing protocols (e.g., constant head rotations) or a more complex model of slow-phase eye velocity than that presented in Eq. 3. For example, Eq. 7 accounts for a phase shift in the velocity input to the integrator, yet remains in the form of a linear regression (unlike the mathematically equivalent expression used in Eq. 8). Higher order terms could be added to account for nonlinear dependence on eye position or head velocity. Galiana et al. (1995), using a similar linear regression technique to characterize the VOR in normal animals ( $k$ assumed to be 0 ), found that some eye velocity data is fit better using a cubic function of head velocity. We have found that saturation effects, commonly seen in animals with an unstable integrator $(k>0)$, can be reasonably accounted for by including a cubic function of eye position (G. Major, R. Baker, and D. W. Tank, unpublished observations). Similarly, a model that additionally fits eye acceleration or higher derivative data could, in principle, be accommodated by the linear regression method. However, we suspect that noise in the computation of higher derivatives would limit the utility of this method for such models.

The fitting method presented here should be more generally applicable to VOR data taken at low head velocities for which the combined contribution of the direct pathway and eye plant is negligible (and with post-saccadic drift regions of the eye position data removed). For example, we expect that it should be applicable to high-frequency, low-amplitude data for which the head velocity is low. The data analyzed here (Kaneko 1999) were recorded at fixed amplitude, so that higher frequency data correspond to proportionally higher head velocities. To fit all portions of higher head velocity data correctly requires a two-stage model of the oculomotor neural integrator, with an oculomotor plant driven by both a neural integrator and a direct pathway. Applying our fitting technique only to intervals of high-frequency data for which head velocity is low, however, did give integrator parameter values consistent across frequencies (data not shown).

The non-phase-lagged response to a sinusoidal input observed in the presence of saccades (Fig. 2B) is more generally an example of the response of a low-pass filter with sharp resets. Similar behavior has been noted in the ability of a leaky integrate-and-fire neuron to track a sinusoidal input without significant phase lag (Knight 1972). Linear systems techniques, such as Fourier analyses and phase-shift methods, cannot be applied to such systems because they neglect the nonlinearity introduced by the resets. The fitting method presented here should be useful in analyzing these systems because it allows the linear filter behavior of the system between resets to be isolated from the nonlinear contributions of the resets themselves.

In conclusion, we have shown how phase-shift analyses of the VOR can lead to erroneous estimations of the neural integrator time constant because they neglect the effect on eye velocity of saccadic resets of eye position (Galiana 1991; Mettens et al. 1994). Because our results are based upon a lesion study, we cannot rule out the possibility of two functionally separate but anatomically co-localized integrators residing in the area (nph) affected by the lesions. However, our results do suggest a common role for nph as a substrate of neuronal integration for both saccadic and vestibular input.

We thank R. G. Baker and L. F. Abbott for helpful discussions.

This work was supported by the Howard Hughes Medical Institute, National Eye Institute Grant 06558, Division of Research Resources Grant 00166, and National Science Foundation Grant 9986022.

\section{REFERENCES}

CANNON SC AND RobINSON DA. Loss of the neural integrator of the oculomotor system from brain stem lesions in monkey. J Neurophysiol 57: 13831409, 1987.

Cheron G And Godaux E. Disabling of the oculomotor neural integrator by kainic acid injections in the prepositus-vestibular complex of the cat. J Physiol 394: 267-290, 1987.

Cheron G, Godaux E, Laune JM, and Vanderkelen B. Lesions in the cat prepositus complex: effects on the vestibulo-ocular reflex and saccades. J Physiol 372: 75-94, 1986.

FUCHS AF AND KIMM J. Unit activity in vestibular nucleus of the alert monkey during horizontal angular acceleration and eye movement. J Neurophysiol 38: 1140-1161, 1975.

GaLiana HL. A nystagmus strategy to linearize the vestibulo-ocular reflex. IEEE Trans Biomed Eng 38: 532-543, 1991.

GALIANA HL AND OUTERBRIDGE JS. A bilateral model for central neural pathways in vestibuloocular reflex. J Neurophysiol 51: 210-241, 1984.

Galiana HL, Smith HL, and Katsarkas A. Comparison of linear vs. nonlinear methods for analysing the vestibulo-ocular reflex (VOR). Acta Otolaryngol 115: 585-596, 1995.

KANEKO CRS. Eye movement deficits after ibotenic acid lesions of the nucleus prepositus hypoglossi in monkeys. I. Saccades and fixation. J Neurophysiol 78: 1753-1768, 1997.

KANEKO CRS. Eye movement deficits following ibotenic acid lesions of the nucleus prepositus hypoglossi in monkeys. II. Pursuit, vestibular, and optokinetic responses. J Neurophysiol 81: 668-681, 1999.

KNIGHT BW. Dynamics of encoding in a population of neurons. J Gen Physiol 59: 734-766, 1972.

Mettens P, Godaux E, Cheron G, and Galiana HL. Effect of muscimol microinjections into the prepositus hypoglossi and the medial vestibular nuclei on cat eye movements. J Neurophysiol 72: 785-802, 1994.

PRECHT W. Vestibular system. In: Neurophysiology, edited by Hunt C. London: MTP International Review of Science, 1974, p. 81-149.

Rey CG and Galiana HL. Transient analysis of vestibular nystagmus. Biol Cybern 69: 395-405, 1993.

RoBINSON DA. Oculomotor control signals. In: Basic Mechanisms of Ocular Motility and Their Clinical Implications, edited by Lennerstrand G and Bachy-Rita P. New York: Pergamon, 1975, p. 337-374.

Robinson DA. Control of eye movements. In: Handbook of Physiology. The Nervous System. Visual Motor Control, edited by Desmedt JE. Bethesda, MD: Am. Physiol. Soc., 1981, p. 1275-1320.

RoBINSON DA. Integrating with neurons. Annu Rev Neurosci 12: 33-45, 1989. Schneider E, Glasauer S, AND Dieterich M. Central processing of human ocular torsion analyzed by galvanic vestibular stimulation. Neuroreport 11 : $1559-1563,2000$. 